\title{
High Fidelity Simulation in Undergraduate Medical Curricula: Experience of Fourth Year Medical Students
}

\author{
Kodikara, K.G., Karunaratne, W.C.D. \& Chandratilake, M.N.
}

\begin{abstract}
Introduction: Application of theoretical knowledge to management of critically ill patients is a challenging task for medical undergraduates where opportunities to learn clinical skills with regard to management of emergencies are few. High fidelity simulation (HFS) is widely used globally to learn clinical skills in a safe environment. However, research in use of HFS in developing countries such as Sri Lanka is minimal.
\end{abstract}

Purpose: The purpose of this study was to explore the response of medical undergraduates to a high fidelity simulator in the context of management of emergencies.

Method: A pilot group of 30 fourth year medical students underwent a high fidelity simulator session. They completed a self-administered evaluation, which included both open and close ended questions and participated in a focus group discussion post-simulation. Descriptive statistics were employed to analyze the responses to close-ended questions and the responses to open- ended questions and focus group discussion were analyzed for recurring themes.

Result: Students rated the simulation-based learning experience with high positivity. The selfcompetency of $29(96.6 \%)$ students had increased post-simulation while providing a safe learning environment to all students. Majority $(63.3 \% ; n=19)$ felt it helped put theory into practice. Qualitative data gathered from open-ended questions and focus group discussions further confirmed these findings.

Conclusion: The study demonstrates that HFS can be used to provide a safe environment and a satisfying educational experience for students to learn medical emergencies, and as an educational opportunity of translating theory into practice. Although HFSs are costly they appear to be cost effective in terms of educational outcomes.

Keywords: Simulation; medical students; high fidelity; competency

\section{Introduction}

Medical students learn clinical skills related to management of emergencies during their clerkships mainly via peripheral participation and observation (Gordon et al., 2001).

Department of Medical Education, Faculty of Medicine, University of Kelaniya, Sri Lanka

Corresponding Author:

Dr. K.G. Kodikara

Department of Medical Education, Faculty of Medicine, University of Kelaniya, P.O. Box 6, Thalagolla Road, Ragama, Sri Lanka.

Email: *k.kodikara@kln.ac.lk
However, involvement in such experiences are invaluable during the undergraduate years where integration of theory and practice is necessary for the development and honing of clinical skills required of graduating doctors. Young doctors experience significant stress and anxiety particularly during the internship year where they first come into contact with and be responsible for acutely ill patients (Gordon et al., 2001). In such instances, novice doctors may occasionally cause preventable injuries to their patients although they are under close supervision (Ziv et al., 2003). Therefore, developing the clinical skills required to function effectively within a clinical team and learning to approach problems systematically must be learned during the undergraduate years to 
reduce the risk of preventable medical errors. Simulation-based medical education (SBME) has been identified as an effective means of mitigating these issues.

Simulation-based training in emergency management has been widely adopted globally in the post-graduate medical education as well as in undergraduate medical curricula (Morgan \& Cleave-Hogg, 2002; Weller et al., 2003). Originally built to train anaesthesiologists in critical care management, simulators have developed from low technology, low fidelity simulators to advanced, sophisticated high fidelity mannequins. These high fidelity simulators combine a full body mannequin and a sophisticated computer control system which provides various physiological parameters. Pulse rate, respiratory rate, auscultatory sounds and blood pressure can be emitted from the manikin along with various other physical signs including physiologic changes to drug therapy (Maran \& Glavin, 2003; Bradley, 2006). Thus, the students experience a clinical condition with real-time changes in vital parameters which is very realistic in nature. Hence, the students are given the opportunity to learn clinical skills with trial and error, without imposing any risk to the patients or themselves. Literature provides ample evidence globally on the role of high fidelity simulation-based learning in improving knowledge and skills of learners in the management of emergencies (O'flynn \& Shorten, 2009; Okuda et al., 2009).

However, Sri Lanka has lagged behind other countries in incorporating simulation-based learning in undergraduate medical curricula, which could partly be due to high costs associated with HFS. The clinical skills laboratory of the Faculty of Medicine, University of Kelaniya was recently equipped with a high fidelity simulator. As there is currently little evidence into the use of high fidelity simulation among medical undergraduates in Sri Lanka, this study aims to evaluate the response of Sri Lankan medical undergraduates to high fidelity simulator-based training experience in developing the skills of managing medical emergencies, the first study to be published in the Sri Lankan context.

\section{Methods}

The simulation-based training on the management of medical emergencies was piloted among 30 volunteering fourth-year medical students of the Faculty of Medicine, University of Kelaniya. They had already undergone twelve-week clerkships in medicine and had attended the emergency treatment unit, medical wards and lectures in management of common medical emergencies before the commencement of the simulation sessions. The students participated in training on the management of two common medical emergencies at the clinical skills laboratory as small groups of six. Each group underwent twohour sessions per-scenario. The participating students were allowed to withdraw from the sessions at any time. Students were reassured that they were not expected to perform beyond their abilities, and their performance would not affect their assessments. A third generation ultra-high fidelity full body adult simulator was used in these sessions.

Students underwent an initial session of familiarization with the simulator and the equipment. Two common medical emergencies were presented to the students: anaphylaxis and hypovolaemic shock. The students were presented with the problem by the facilitator following which students were given time to plan their responses. The facilitator observed while the students managed the emergencies as groups of six. The facilitator assisted the students when in need, acting as a nurse, thereby preventing inappropriate treatment or prompting initiation of necessary treatment. This prevented simulator death as simulator death could be stressful for the students and could affect the learners negatively. The scenarios were followed by a debriefing session where the students clarified any concerns.

The students responded to a paper-based selfadministered questionnaire at the end of the session which included two parts: Part 1 included items on student perceptions on the simulation-based training activity using a rating scale from 1-5 (1=strongly disagree and $5=$ strongly agree). The items explored the safety of the learning environment, working as a team, improvement in self-confidence to manage similar situations during practice, application of theory into practice, improvement in knowledge and skills on managing medical emergencies and overall views on simulationbased training. The students rated their opinion on the use of simulation as an assessment tool using a rating scale from 1-3 (1=No, 2=Not sure, $3=$ Yes). Students also rated their level of confidence in dealing with medical emergencies before and after the simulationbased learning session on a scale of 1-5, where $1=$ beginner and 5 =master. Part 2 of the questionnaire included open-ended questions with general written comments which sought their opinion on the use of simulation-based 
learning in undergraduate medical education. Finally, a focus group discussion was conducted post-simulation with 12 medical students to further explore the value of high fidelity simulation in their education.

The data gathered from the questionnaire were analyzed using descriptive statistics. The openended questions and data gathered from the focus group discussion were coded and analyzed for recurring themes.

\section{Results}

All 30 students participated in the simulated sessions and responded to the questionnaire. Students rated the simulation-based learning session with the use of high fidelity simulation very positively (Table 1). The students felt safe in the simulated learning environment and indicated that the session helped to improve their knowledge and skills with regard to management of medical emergencies.

Table 1: Students' responses to the questionnaire

\begin{tabular}{|c|c|c|c|c|}
\hline & Question & Median & $\begin{array}{l}\text { Inter- } \\
\text { quartile } \\
\text { range } \\
\text { (IQR) }\end{array}$ & Range \\
\hline a) & Was the learning environment safe? & 4 & $4-5$ & $4-5$ \\
\hline b) & Were you encouraged to work as a team? & 5 & $4-5$ & $4-5$ \\
\hline c) & $\begin{array}{l}\text { Did the session improve your confidence to participate in the } \\
\text { clinical setting? }\end{array}$ & 5 & $4-5$ & $4-5$ \\
\hline d) & Did the session helped you to put theory into practice? & 5 & $4-5$ & $4-5$ \\
\hline e) & $\begin{array}{l}\text { Did the session improve your knowledge on management of } \\
\text { medical emergencies? }\end{array}$ & 5 & $4-5$ & $3-5$ \\
\hline f) & $\begin{array}{l}\text { Did the session improve your skills on management of } \\
\text { emergencies? }\end{array}$ & 5 & $4-5$ & $4-5$ \\
\hline g) & Was the simulation session a valuable experience? & 5 & $4-5$ & $4-5$ \\
\hline
\end{tabular}

1=Strongly disagree, 2=Disagree, 3=Neither agree nor disagree, 4=Agree, 5=Strongly agree

Twenty eight (93.3\%) students thought high fidelity simulation provided an accurate measurement of their ability in managing an acutely ill patient. Most students felt simulation should be included in the formal end of year assessments (Table 2).

Twenty nine (96.6\%) students felt an increase in their self-competency with regard to the management of medical emergencies following the simulation-based learning session where they rated competency with 1=beginner to $5=$ master. Nine $(30.0 \%)$ students felt their competency level increased from level 2 to level 3 , while six $(20.0 \%)$ students felt their competency level increased from level 3 to level 4. 
Table 2: Students' opinion on using simulation as an assessment tool

\begin{tabular}{llcc}
\hline \multicolumn{1}{c}{ Simulation-based assessment } & Yes (\%) & No (\%) & Not sure (\%) \\
\hline Should be included in formal assessments & $21(70.0)$ & $2(6.6)$ & $7(23.3)$ \\
\hline Accurate measure of ability & $28(93.3)$ & $1(3.3)$ & $1(3.3)$ \\
\hline
\end{tabular}

Seventeen (56.6\%) students felt the competency level increase by one level while twelve $(40.0 \%)$ students felt an increase in competency by two levels. Only 1 student indicated that the competency level did not change with the simulation session. Table 3 illustrates the change in competency following the simulation-based learning sessions.

All the students responded enthusiastically to the open-ended questionnaire. With regard to the use of simulation in undergraduate medical curricula, all students were positive with comments such as "excellent", "interesting", "very good" and "wonderful". Table 4 shows the students' opinion on the uses of simulationbased learning on their education.

Twenty five (83.3\%) students indicated simulation should be included more in their education typified by the comments "All medical students should be given this opportunity to learn using this simulator all throughout the five years". Sixteen $(53.3 \%)$ students suggested simulation to be made mandatory in the curriculum. The realism of the high fidelity simulation was prominent with 17 (56.6\%) students indicating the session as a realistic experience. Students valued the opportunity to receive hands-on experience, "We have seen it being done, but we as medical students never get the chance to do this" and "it really made us think and felt the urgency of the situation, which I never felt before". Most students identified simulation as a valuable practice opportunity to prepare for internship: "We are supposed to manage sick patients when we pass-out but I never got that kind of training until now".

The post-simulation focus group conducted with participants, putting theory into practice in a safe environment emerged as the main advantage of high fidelity simulation. They identified cost as a disadvantage while $83.3 \%$ felt the lack of trained staff as a major barrier in implementing regular HFS sessions at the Faculty.

Table 3: Change of competency levels after simulation-based training

\begin{tabular}{ccccc}
\hline \multirow{2}{*}{$\begin{array}{c}\text { Competency level } \\
\text { before }\end{array}$} & $\mathbf{4}$ & $\mathbf{2}$ & $\mathbf{3}$ & $\mathbf{4}$ \\
\cline { 2 - 5 } $\mathbf{1}(\mathrm{N}=7)$ & 0 & $2(28.6 \%)$ & $5(71.5 \%)$ & 0 \\
\hline $\mathbf{2}(\mathrm{N}=17)$ & 0 & $\begin{array}{c}1(5.9 \%) \\
\text { (unchanged) }\end{array}$ & $9(53 \%)$ & $7(41.1 \%)$ \\
\hline $\mathbf{3}(\mathrm{N}=6)$ & 0 & 0 & 0 & $6(100 \%)$ \\
\hline $\mathbf{4}(\mathrm{N}=0)$ & 0 & 0 & 0 & 0 \\
\hline
\end{tabular}


Table 4: The students' opinion on use of simulation in undergraduate medical education

\begin{tabular}{|c|c|c|c|}
\hline \multicolumn{2}{|c|}{ Written comment groupings } & \multicolumn{2}{|c|}{ Student responses } \\
\hline Theme & Subcategory & $\mathbf{n}$ & $\%$ \\
\hline \multirow[t]{2}{*}{ Overall assessment } & Very good & 24 & 80.0 \\
\hline & Interesting & 18 & 60.0 \\
\hline \multirow[t]{4}{*}{ Pedagogic utility } & Good learning tool & 11 & 36.6 \\
\hline & Motivate learning & 6 & 20.0 \\
\hline & Promote critical thinking and reasoning & 13 & 43.3 \\
\hline & Apply theory into practice & 19 & 63.3 \\
\hline \multirow[t]{2}{*}{ Process descriptors } & Realistic experience & 17 & 56.6 \\
\hline & Hands on experience & 23 & 76.6 \\
\hline \multirow[t]{3}{*}{ Approach considerations } & Systematic approach to a problem & 9 & 30.0 \\
\hline & Need to know the basics & 14 & 46.6 \\
\hline & Need to organize response & 4 & 13.3 \\
\hline \multirow[t]{3}{*}{ Future goals } & Need more sessions & 25 & 83.3 \\
\hline & Make it mandatory & 16 & 53.3 \\
\hline & Practice for internship & 21 & 70.7 \\
\hline
\end{tabular}

\section{Discussion}

In this study, the students rated the simulationbased learning using a high fidelity simulator as a valuable experience. The students were stimulated by the realistic experience to apply theoretical knowledge into practice. Students felt their self-assessed competency improve with simulation-based learning session and appeared to have increased level of confidence to deal with medical emergencies. They wanted more exposure with simulation-based learning sessions and felt simulation as good practice for internship. It helps students to improve both knowledge and skills, complying with similar studies. Studies conducted previously among 
medical students have found that students value simulation-based leaning highly (Morgan and Cleave-Hogg, 2000; Gordon et al., 2001; Weller et al., 2004). Our study confirmed their findings and confirmed that the positive attributes of simulation-based medical education transcends different institutions and countries across the world. However, it was interesting to note that the use of HFS in the Southeast Asian context was not observed to the best of our attempts.

Simulation has been widely used in fields such as aviation and space industries, where high fidelity simulation has been a standard tool of training for both technical and non-technical skills (Bradley, 2006). The learning processes identified by the participants during the high fidelity simulation training are considered as more effective than traditional learning methods and were consistent with known principles of effective learning (Kaufman, 2003), which validate the findings of this study. Solving reallife problems, providing opportunities to practice, facilitating reflection are a few principles proposed by Kaufman (2003) as a guide to effective teaching/learning practice. Although these principles are applicable to many teaching contexts, simulation-based medical education harnesses all these principles with ease, thereby emerging as a highly effective and an innovative learning tool. Students' positive experience may have attributed to this comprehensiveness of the educational experience.

Despite evident pedagogical advantages of high fidelity simulation-based learning, implementation of high fidelity simulation in undergraduate medical curricula in countries such as Sri Lanka is limited. This could well be due to the high costs associated with high fidelity simulators. One may argue that when patients are in abundance for medical education, investing on HFS is not cost effective. However, in developing the competence among students on dealing with medical emergencies and with growing concerns protecting patient rights, HFS has become a requirement of the hour. The growing evidence on the use of HFS suggests that it is cost-effective in terms of achieving educational outcomes. Although robust research is required to evaluate the efficacy of high fidelity simulation, i.e. whether there is real impact on real practice, our study suggests that high fidelity simulation in undergraduate curricula could be a powerful learning tool in developing at least certain clinical competencies.

\section{Limitations}

Our study has several limitations. A significant limitation of this study might be that it involved a relatively small group of students from a single year of study within a single institution. A larger sample across students involved in clinical teaching/learning might produce further insights into learner-centered, high-fidelity simulation-based training for improving medical students performance in management of medical emergencies.

It is possible that the self-rating scores may increase and result in overconfidence of students with high fidelity simulators due to novelty of the simulator and the simulated learning experience (Massoth et al., 2019). Therefore, we attempted to minimize this effect through a period of familiarization with the simulators prior to the scenarios. Increased student exposure to simulation could address this problem, but were not feasible within the time constraints of the student curriculum. Although an increase in self-confidence and competence was demonstrated in this study, more research is required to evaluate the retention of the acquired skill in the long-term.

Although there is no doubt that the real patient is the ideal for the medical student, the advantages of high fidelity simulation in learning outweighing the risks to patients makes HFS an ideal educational tool. However, it is widely demonstrated that HFS is not superior to low-fidelity simulators (Dillon et al., 2004), and in view of the cost and resource intensiveness of HFS, the feasibility of utilizing low-fidelity simulators where possible need be encouraged especially in resource poor countries such as Sri-Lanka.

Despite these limitations, our study demonstrates that medical students embraced the HFS driven learning experience and complying with similar researches, proves that $\mathrm{HFS}$ is an ideal educational tool which facilitates student learning where there is minimal exposure and maximum risk to the patient.

\section{Conclusion}

This study demonstrated that medical students felt high fidelity simulation-based learning as a valuable educational experience. Simulationbased learning allows students to practice in a safe learning environment and provides an opportunity to integrate theoretical knowledge and practice while improving both self- 
confidence and competence. However, this study was limited to the students' selfassessment and their opinions. More research is needed to explore the impact on learning and skill transfer to real practice setting to justify the cost of implementing high fidelity simulationbased learning in undergraduate medical curricula, especially in Southeast Asian context.

\section{Conflict of Interest}

The authors declare no conflict of interest.

\section{Ethics Committee Approval}

As this was an educational evaluation, ethics approval was not requested.

\section{References}

Bradley, P. (2006) 'The history of simulation in medical education and possible future directions', Medical Education, 40, 3, pp. 254-262.

Dillon, G. F., Boulet, J., Hawkins, R. E. \& Swanson, D. B. (2004) 'Simulations in the United States Medical Licensing', Quality and Safety in Health Care, 13, 1, November, pp. 41-45.

Gordon J, Wilkinson W, Shaffer D.W. \& Armstrong, E.G. (2001) 'Practising medicine without risks: students and educators responses to high fidelity patient simulation', Academic Medicine, 6, 5, pp. 469-472.

Kaufman, D. M. (2003) 'ABC of learning and teaching in medicine: Applying educational theory in practice', BMJ, 326, pp. 213-216.

Maran, N.J. \& Glavin, R.J., 2003. Low-to high-fidelity simulation-a continuum of medical education?. Medical education, 37, pp.22-28.
Massoth, C., Röder, H., Ohlenburg, H., Hessler, M., Zarbock, A., Pöpping, D.M. \& Wenk, M., 2019. High-fidelity is not superior to low-fidelity simulation but leads to overconfidence in medical students. BMC medical education, 19, 1, p.29.

Morgan, P.J. \& Cleave-Hogg, D., 2000. A Canadian simulation experience: faculty and student opinions of a performance evaluation study. British Journal of Anaesthesia, 85, 5, pp.779-781.

Morgan, P. J. \& Cleave-Hogg, D. (2002) 'A worldwide survey of the use of simulation in anesthesia', Canadian Journal of Anesthesia, 49, 7, pp. 659662.

O'flynn, S. \& Shorten, G. (2009) 'Editorial: Simulation in undergraduate medical education', European Journal of Anaesthesiology, 26, 2, pp. 93-95.

Okuda, Y., Bryson, E. O., DeMaria, S., Jacobson, L., Quinones, J., Shen, B. \& Levine, A. I. (2009) 'The utility of simulation in medical education: What is the evidence?', Mount Sinai Journal of Medicine, pp. 330-343.

Weller, J., Robinson, B., Larsen, P. \& Caldwell, C. (2004) 'Simulation-based training to improve acute care skills in medical undergraduates', New Zealand Medical Journal, 117, 1204, pp. 1-8.

Weller, J., Wilson, L. \& Robinson, B., 2003. Survey of change in practice following simulation-based training in crisis management. Anaesthesia, 58, 5, pp.471-473.

Ziv, A., Wolpe, P. R., Small, S. D. \& Glick, S. (2003) 'Simulation-based medical education: an ethical imperative.' Academic medicine: journal of the Association of American Medical Colleges, 78, 8, pp. 